Table 1. IRs of SI in RA and PsA patients starting a TNFi Jan 2009 - Dec 2018. HRs for PsA compared to RA.

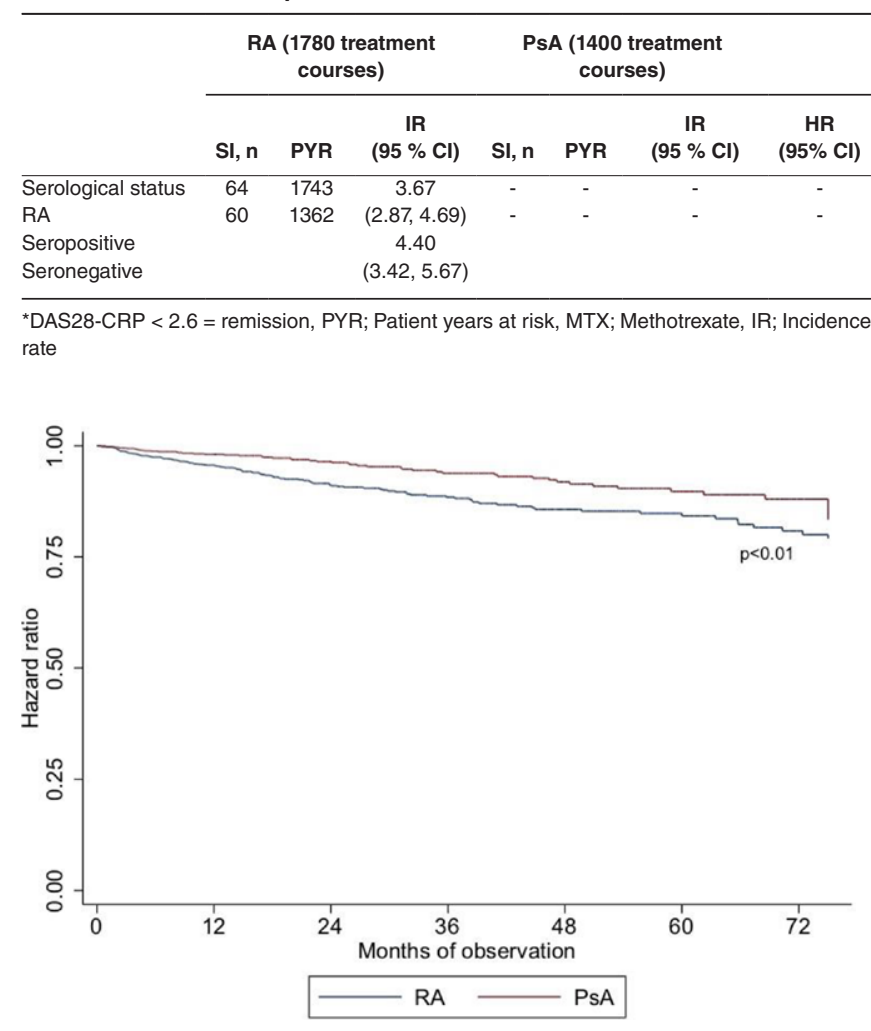

Figure 1. Age- and gender-adjusted risk of SI across RA and PsA

Disclosure of Interests: Ingrid Egeland Christensen: None declared, Siri Lillegraven: None declared, Joe Sexton: None declared, Tore K. Kvien Grant/research support from: Received grants from Abbvie, Hospira/Pfizer, MSD and Roche (not relevant for this abstract)., Consultant of: Have received personal fees from Abbvie, Biogen, BMS, Celltrion, Eli Lily, Hospira/Pfizer, MSD, Novartis, Orion Pharma, Roche, Sandoz, UCB, Sanofi and Mylan (not relevant for this abstract)., Paid instructor for: Have received personal fees from Abbvie, Biogen, BMS, Celltrion, Eli Lily, Hospira/Pfizer, MSD, Novartis, Orion Pharma, Roche, Sandoz, UCB, Sanofi and Mylan (not relevant for this abstract)., Speakers bureau: Have received personal fees from Abbvie, Biogen, BMS, Celltrion, Eli Lily, Hospira/Pfizer, MSD, Novartis, Orion Pharma, Roche, Sandoz, UCB, Sanofi and Mylan (not relevant for this abstract)., Till Uhlig Consultant of: Lilly, Pfizer, Speakers bureau: Grünenthal, Novartis, Sella Aarrestad Provan Consultant of: Novartis

DOI: 10.1136/annrheumdis-2020-eular.4623

\section{AB0761 DEMOGRAPHIC AND CLINICAL FEATURES OF JUVENILE-ONSET PSORIATIC ARTHRITIS: RESULTS FROM PSART-ID REGISTRY}

S. Ergulu Eșmen ${ }^{1}$, O. Bayindir ${ }^{2}$, E. Kasapoğlu ${ }^{3}$, S. Bakırcl ${ }^{4}$, D. Solmaz ${ }^{2}$ G. Kimyon ${ }^{5}$, A. Doğru', E. Dalkılıç , C. Özişler ${ }^{8}$, M. Can $^{3}$, S. Akar², E. F. Tarhan ${ }^{9}$,

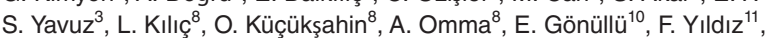

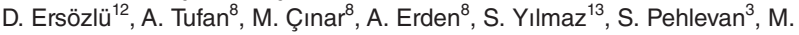
T. Duruöz ${ }^{3}$, S. Aydin ${ }^{14}$, U. Kalyoncu ${ }^{8}{ }^{1} P S A R T$ ID, Kayseri, Kayseri, Turkey; ${ }^{2} P S A R T-I D$, izmir, izmir, Turkey; ${ }^{3}$ PSART-ID, istanbul, istanbul, Turkey; ${ }^{4} P S A R T-I D$, Antalya, Antalya, Turkey; ${ }^{5}$ PSART-ID, Hatay, Hatay, Turkey; ${ }^{6}$ PSART-ID, Isparta, Isparta, Turkey; ${ }^{7} P S A R T-I D$, Bursa, Bursa, Turkey; ${ }^{8} P S A R T-I D$, Ankara, Ankara, Turkey; ${ }^{9}$ PSART-ID, Muğla, Muğla, Turkey; ${ }^{10}$ PSART-ID, Sakarya, Sakarya, Turkey; ${ }^{11}$ PSART-ID, Kahramanmaraş, Kahramanmaraş, Turkey; ${ }^{12} P S A R T-I D$, Adana, Adana, Turkey; ${ }^{13}$ PSART-ID, Konya, Konya, Turkey; ${ }^{14}$ PSART-ID, Ottawa, Ottawa, Canada

Background: Although psoriatic arthritis (PsA) may be seen at any decades, juvenil onset PsA is relatively rare. Moreover, there were no more data about clinical features, treatments, and course in juvenile PsA when they reached to adult age.

Objectives: The objective of this study was to assess and compare demographic and clinical features for juvenile onset PsA and adult onset PsA.

Methods: PsART-ID is a multicenter, international database, investigating the disease characteristic in real life (1). Briefly, demographic data, PsA subtypes, uveitis, enthesitis, dactylitis, Co-morbidities, disease activity scores (TJC, SJC, VAS-pain, VAS patients and physician global assessments, VAS-fatigue, BASDAI), and functional status (HAQ-DI, BASFI) were recorded. Psoriasis and PsA starting age were noted, as well. Patients were classified as juvenile PsA or juvenile PsO (under 18 years old). Results were compared regarding to juvenile versus adult onset age.

Results: Overall, 1644 PsA patients were included to study, 301/1644 (18.3\%) patients had juvenile onset psoriasis. Of 39/1644 (2.4\%) patients had juvenile onset PsA, as well. As expected, juvenile onset PsA patients were younger, however PsA disease duration were longer than adult onset PsA patients. There were no any difference between demographic and clinical data, except BMI and enthesitis were less frequently at the juvenile onset PsA groups. Although, ever csDMARD using were similar between two groups, however, juvenile onset PsA patients were used more frequently bDMARDs.

Table. Comparison of demographic and clinical characteristics of juvenile and adult-onset psoriatic arthritis

\begin{tabular}{lccc}
\hline & Juvenile onset & Adult onset & $\mathbf{p}$ \\
\hline $\mathrm{N}(\%)$ & $39(2.4)$ & $1605(97.6)$ & \\
Female Sex n (\%) & $24(61.5)$ & $1006(62.7)$ & 0.884 \\
PsA beginning age mean (SD) & $13.3 \pm 3.85$ & $42.3 \pm 12.9$ & $<0.001$ \\
Current age mean (SD) & $26.6 \pm 10.7$ & $47.3 \pm 13.07$ & $<0.001$ \\
Duration of psoriasis (years) & $17.10 \pm 11.26$ & $14.75 \pm 11.78$ & 0.124 \\
Duration of psoriatic arthritis (years) & $13.5 \pm 11$ & $5.06 \pm 6.7$ & $<0.001$ \\
Cigarette smoking (ever) n (\%) & $15 / 38$ & $641 / 1494$ & 0.72 \\
Education duration/year (mean,SD) & $10.09 \pm 3.67$ & $9.52 \pm 4.81$ & 0.464 \\
BMl (kg/m2) (mean, SD) & $24.5 \pm 5.1$ & $28.3 \pm 5.21$ & $<0.001$ \\
Family history of PsO/PsA n (\%) & $15(38.5)$ & $559(34.9)$ & 0.642 \\
Nail involvement n (\%) & $18(46.2)$ & $762(47.5)$ & 0.864 \\
Dactilitis n (\%) & $9(23.7)$ & $367(24)$ & 0.958 \\
Entesitis n (\%) & $3(7.9)$ & $384(25.7)$ & 0.013 \\
Uveitis n (\%) & - & $13(4.3)$ & 0.713 \\
Axial involvement (\%) & $15(38.5)$ & $464(29)$ & 0.199 \\
Methotrexate & $36(92.3)$ & $1348(84)$ & 0.162 \\
Sulfasalazine & $17(43.6)$ & $612(38.1)$ & 0.488 \\
Leflunomide & $14(35.9)$ & $379(23.6)$ & 0.076 \\
Biologic DMARDs & $102(33.9)$ & $358(26.8)$ & 0.013 \\
& & &
\end{tabular}

Conclusion: Although psoriasis may be seen frequently in the juvenile age, juvenile onset PsA was not so frequent in our PsA cohort. Although, ever csDMARD using were similar between two groups, however, juvenile onset PsA patients were used bDMARDs more frequently.

References:

[1] Kalyoncu U et al. The Psoriatic Arthritis Registry of Turkey: results of a multicenter registry on 1081 patients. Rheumatology. 2017;56:279-286.

Disclosure of Interests: Serpil ERGULU EŞMEN: None declared, Ozun Bayindir: None declared, esen kasapoğlu: None declared, Sibel Bakırcı: None declared, Dilek Solmaz: None declared, Gezmiş Kimyon: None declared, Atalay Doğru: None declared, Ediz Dalkılıç: None declared, Cem Özişler: None declared, Meryem Can: None declared, Servet Akar: None declared, Emine Figen Tarhan: None declared, Sule Yavuz: None declared, Levent Kıllç: None declared, Orhan Küçükşahin: None declared, Ahmet Omma: None declared, Emel Gönüllü: None declared, Fatih Yıldız: None declared, Duygu Ersözlü: None declared, abdurrahman tufan: None declared, Muhammet Çınar: None declared, Abdulsamet Erden: None declared, Sema Yılmaz: None declared, Seval Pehlevan: None declared, Mehmet Tuncay Duruöz: None declared, Sibel Aydin: None declared, Umut Kalyoncu Consultant of: Abbvie, Amgen, Janssen, Lilly, Novartis, UCB

DOI: 10.1136/annrheumdis-2020-eular.520

\section{AB0762 METABOLIC SYNDROME IN EGYPTIAN PSORIATIC ARTHRITIS PATIENTS.}

M. Imam ${ }^{1}$, M. Helal ${ }^{1}$, M. Hassan ${ }^{1}$, E. Hassan ${ }^{2}$, S. Essam ${ }^{3} .{ }^{1}$ Faculty of Medicine, Alexandria University, Rheumatology and Rehabilitation, Alexandria, Egypt;

${ }^{2}$ Alexandria university, Internal medicine, Alexandria, Egypt; ${ }^{3}$ Faculty of Medicine, Alexandria University, Alexandria, Egypt

Background: Psoriatic arthritis (PSA) is a chronic inflammatory arthritis affecting $20: 30 \%$ of patients with skin Psoriasis (PsO). It is strongly associated with obesity, particularly excess visceral adiposity, which leads to insulin resistance, hyperglycemia, dyslipidemia, and hypertension (HTN).

All the previous findings are grouped in Metabolic Syndrome (MetS) which increase the risk of development of Type 2 diabetes mellitus (T2DM) by five folds and cardiovascular disease (CVD) by two folds.

Objectives: The aim of this work was to define those who fulfill MetS criteria in PsA patients thus of greater risk to develop CVD and T2DM.

Methods: Fifty PsA patients diagnosed according to the CASPAR criteria and 50 matched healthy controls were included in this study. 